\title{
ANÁLISE DA EFICIÊNCIA DOS FUNDOS DE INVESTIMENTO IMOBILIÁRIO EM UM PERÍODO DE CRISE ECONÔMICA
}

\section{RESUMO}

O Plano Real de estabilização ensejou grandes transformações no setor financeiro do país e abriu espaço para o desenvolvimento de formas alternativas de aplicações de recursos. Os fundos caracterizados como renda variável e multimercado têm sido retratados em vários estudos acadêmicos, mas persiste uma carência em relação aos Fundos de Investimento Imobiliário (FII), que são lastreados em ativos da construção civil. Este artigo objetiva sanar em parte esta lacuna ao analisar o desempenho de FII em um período de retração econômica. A amostra contempla quarenta e quatro fundos registrados na Comissão de Valores Mobiliários (CVM) e que apresentaram cotações diárias no período de agosto de 2013 a setembro de 2016. A abordagem é quantitativa, tendo sido empregados indicadores econômicofinanceiros (índices de Sharpe e Sortino), análise de regressão (Alfa de Jensen) e o modelo de otimização Data Envelopment Analysis - DEA. Os resultados não foram favoráveis aos FIIs como alternativa atraente para a aplicação de recursos no período analisado. Considerando-se o risco e retorno, poucos fundos conseguiram superar o benchmark de mercado. Os procedimentos metodológicos permitiram, ainda, verificar a eficiência relativa de cada fundo, constatando-se que houve perda de eficiência por parte significativa da indústria; 28 tornaram-se menos eficientes, 12 não apresentaram mudanças dignas de notas, e apenas 4 elevaram seus níveis de eficiência. Ademais, os resultados obtidos com o emprego dos vários modelos sinalizam diferentes possibilidades de análise e podem contribuir para a tomada de decisão por parte dos gestores de carteiras e investidores do mercado de capitais.

Palavras-chave: Fundos Imobiliários. Análise de Eficiência. DEA.

\section{EFFICIENCY ANALYSIS OF REAL ESTATE INVESTMENT FUNDS IN A PERIOD OF ECONOMIC}

\section{ABSTRACT}

The Real Plan of stabilization brought about great transformations in the financial sector of the country and paved the way for the development of alternative forms of resource applications. The funds characterized as variable income and multimarket have been analyzed in several academic studies, but there is still a lack of concern with the Real Estate Investment Funds (FII), which are backed by civil construction assets. This article aims at partially remedying this gap by analyzing the performance of FII in a period of economic downturn. The sample includes forty-four funds registered at Comissão de Valores Mobiliários (CVM) that presented daily quotations from August 2013 to September 2016. The approach is quantitative, using economic and financial indicators (Sharpe and Sortino indexes), regression analysis (Alfa de Jensen) and the Data Envelopment Analysis (DEA) optimization model. The results were not favorable to the FIIs as an attractive alternative to the application of resources in the analyzed period. Taking into account the risk and return, few funds were able to surpass the market benchmark. The methodological procedures made also possible to verify the relative efficiency of each fund leading to the conclusion that there was a loss of efficiency in a significant part of the industry; 28 became less efficient, 12 did not present changes worthy of mention, and only 4 increased their levels of efficiency. In addition, the results obtained with the use of the various models bring about different possibilities of analysis and can contribute to the decision-making by portfolio managers and investors in the capital market.

Keywords: Real Estate Funds. Efficiency Analysis. DEA. 


\section{ANÁLISIS DE LA EFICIENCIA DE LOS FONDOS DE INVERSIÓN INMOBILIARIO EN UN PERÍODO DE CRISIS ECONÓMICA}

\section{RESUMEN}

El Plan Real de estabilización ha generado grandes transformaciones en el sector financiero del país y ha abierto espacio para el desarrollo de formas alternativas de aplicaciones de recursos. Los fondos caracterizados como renta variable y multimercado han sido retratados en vários estudios académicos, pero persiste una carencia en relación a los Fondos de Inversión Inmobiliario (FII), que son respaldados en activos de la construcción civil. Este artículo tiene por objeto sanar en parte esta lacuna al analizar el desempeño de FII en un período de retracción económica. La muestra contempla cuarenta y cuatro fondos registrados en la Comissão de Valores Mobiliários (CVM) y que presentaron cotizaciones diárias en el período de agosto de 2013 a septiembre de 2016. El abordaje es cuantitativo, habiendo sido empleados indicadores económico-financieros (índices de Sharpe y Sortino), análisis de regresión Alfa de Jensen) y el modelo de optimización Data Envelopment Analysis - DEA. Los resultados no fueron favorables a los FII como alternativa atractiva para la aplicación de recursos en el período analizado. Considerando el riesgo y el retorno, pocos fondos lograron superar el benchmark de mercado. Los procedimientos metodológicos permitieron, además, verificar la eficiencia relativa de cada fondo, constatando que hubo pérdida de eficiencia por parte significativa de la industria; 28 se han vuelto menos eficientes, 12 no presentaron cambios dignos de billetes, y sólo 4 elevaron sus niveles de eficiencia. Además, los resultados obtenidos con el empleo de los diversos modelos señalan diferentes posibilidades de análisis y pueden contribuir a la toma de decisión por parte de los gestores de carteras e inversores del mercado de capitales.

Palabras clave: Fondos Inmobiliarios. Análisis de Eficiencia. DEA.

\footnotetext{
${ }^{1}$ Doutor em Economia pela University of London, Inglaterra. Professor do Mestrado Profissional em Administração da Fundação Pedro Leopoldo - FPL. Brasil. E-mail: ronaldo.locatelli@yahoo.com.br

${ }^{2}$ Mestre em Administração pelo Programa de Mestrado Profissional em Administração da Fundação Pedro Leopoldo FPL. Brasil. E-mail: thiagoreisconsult@ gmail.com

${ }^{3}$ Doutor em Ciências Econômicas e Empresariais pela Universitat Autònoma de Barcelona, Espanha. Professor do Mestrado Profissional em Administração da Fundação Pedro Leopoldo - FPL. Brasil. E-mail: jedson.lara@hotmail.com

${ }^{4}$ Doutor em Administração pelo Cepead/UFMG. Professor do Curso de Mestrado Profissional em Administração da Fundação Pedro Leopoldo - FPL. Brasil. E-mail: w.ramalho@yahoo.com.br
} 


\section{INTRODUÇÃO}

A partir da estabilização da moeda brasileira, em meados de 1994, observou-se a reestruturação do setor financeiro pavimentadas pelos Programas de Reestruturação e Fortalecimento do Setor Financeiro Nacional (PROER) e de Incentivo à Redução do Setor Público Estadual na Atividade Bancária (PROES), e por novas regras prudenciais estabelecidas pelo Banco Central do Brasil. O aumento de confiança dos investidores e a queda na taxa de juros abriram espaço para o desenvolvimento de diversos produtos financeiros, e os fundos de investimentos se destacaram, ocupando importante papel em carteiras e portfólios. Dentre estes figuram os FII, que transacionam cotas de investimentos com base em ativos da construção civil.

Segundo a ANBIMA (2016), os FIIs exibiram desde 2005 uma forte expansão com um número significativo de novas ofertas amparadas no crescimento da indústria de construção civil em todo o país (Locatelli, Inês, Lara \& Nogueira, 2017). Esta classe de fundos movimenta um patrimônio de cerca de 60 bilhões de reais geridos por algo em torno de 260 empresas. $\mathrm{O}$ grande diferencial está na possibilidade de aproveitar as oportunidades com diversificação de riscos ao investir em diversos imóveis, auferindo a rentabilidade propiciada pela locação, arrendamento, venda do imóvel e demais atividades do setor imobiliário que refletem no valor da cota do fundo.

Apesar das oportunidades oferecidas pelo setor da construção civil, o momento atual representado por uma recessão econômica e caos político impõe uma análise criteriosa na escolha de ativos, que deve ser amparada por teorias e modelos que levam em consideração o trade-off entre risco e retorno. Nesta perspectiva, o presente trabalho tem por objetivo analisar o desempenho dos principais fundos de investimentos imobiliários no Brasil negociado na bolsa de valores no período de setembro de 2013 a agosto de 2016, sob a perspectiva do investidor.

Houve grandes avanços na teoria de finanças que amparam as análises e recomendações para a escolha de carteiras de investimentos com o intuito de reduzir a exposição ao risco e aumentar os retornos. Os estudos de Markowitz (1959), Sharpe (1964) e Lintner (1965) quebraram paradigmas e modernizaram a área, especialmente no que diz respeito à precificação de ativos e seleção de portfólios. Reconhece-se hoje que a junção destas teorias com análise estatísticas apuradas fornece o embasamento para uma tomada de decisão de investimento mais consciente (Elton, Gruber, Brown \& Goetzmann, 2012).

Nesta pesquisa serão quantificados o desempenho e os riscos dos mais importantes fundos de investimentos imobiliários do país mediante o emprego dos Índices de Sharpe e de Sortino e apresentadas estimativas do Alfa de Jensen, tendo em vista o benchmark de mercado. Será empregada também a Análise Envoltória de Dados (DEA), que incorpora outras variáveis na análise. Esta ferramenta de engenharia de produção possibilita a análise de desempenho de cada unidade tomadora de decisão, e tem sido aplicada ao mercado financeiro com resultados interessantes e consistentes (Lopes, Carneiro, Schneider \& Lima, 2010; Iquiapaza \& Fonseca, 2012).

Este estudo está estruturado em cinco seções, contando com esta breve introdução. Na segunda seção são introduzidos os conceitos, as teorias e os instrumentais quantitativos mais adequados para a análise de desempenho relativo de investimentos e de seleção de carteiras. A terceira seção apresenta os procedimentos metodológicos adotados e informações sobre a amostra. A quarta seção é dedicada à discussão dos resultados alcançados. E, finalmente, a quinta seção apresenta as considerações finais, envolvendo reflexões sobre o desempenho dos fundos e aplicabilidade das teorias e instrumentais utilizados na pesquisa.

\section{REFERENCIAL TEÓRICO}

Há uma proliferação de produtos financeiros, o que torna difícil para a população direcionar de forma adequada as economias acumuladas ao longo da vida. O principal objetivo da poupança está em postergar o consumo, visando à manutenção do padrão de vida mesmo após a aposentadoria, o que requer direcionar os recursos para aplicações com retornos condizentes com os riscos assumidos.

O retorno do investimento contempla a variação do preço do ativo, que tem o poder de agregar ou destruir valor dos investidores. Já o risco pode ser entendido como uma medida de volatilidade associada aos retornos esperados de uma decisão de investimento (Berk, Demarzo \& Harford, 2009). O relacionamento entre retorno e risco nas decisões de investimento foi tratado de forma consistente por Markowitz (1959), e resultou no desenvolvimento de um modelo matemático de otimização de carteiras baseado na combinação de ativos individuais para reduzir a volatilidade/risco (desvio padrão dos retornos). Posteriormente, o conceito de risco foi aprimorado, mediante a suposição que o risco total pode ser classificado como específico (diversificável) e risco sistemático ou não diversificável (beta do ativo) (Damodaran, 2016).

\section{Índices de Sharpe, Sortino e Alfa de Jensen}

Baseado na concepção de Markowitz (1959) e Sharpe (1966) desenvolveu um índice que retrata a medida da rentabilidade ajustada pelo risco total do 
portfólio. O Índice de Sharpe (IS) reporta-se a eficiência de um específico portfólio, mediante a

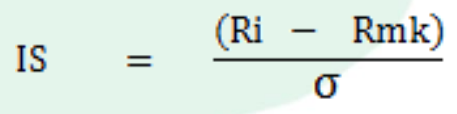

seguinte formulação:

Sendo:

$\mathrm{Ri}=$ retorno médio da carteira;

$\mathrm{Rmk}=$ retorno propiciado pelo benchmark, que é o retorno mínimo aceitável;

$\sigma=$ risco total, medido pelo desvio padrão dos retornos da carteira.

Com base nesta formulação, quanto maior o índice, maior será o retorno por unidade de risco, e consequentemente melhor é o desempenho do fundo. Se o índice apresentar resultado negativo, este terá um desempenho pior que o resultado do benchmark em questão. Varga (2001) intitula o índice de Sharpe como instrumento de investimento zero que visualiza o retorno propiciado pela arbitragem entre algum benchmark e o ativo que está sendo avaliado.
$\mathrm{Na}$ mesma linha teórica, o índice de Sortino também contribuiu para o aprimoramento da comparação de ativos (Sortino \& Price, 1994). O diferencial deste índice é que ele avalia o retorno pelo mínimo risco aceitável pelo investidor, e o seu dimensionador de risco é o dowwside risk, que é exatamente a parte do risco no qual o investidor está realmente preocupado: o desempenho abaixo da meta aceitável (Lima, 2015; Eid, Rochman \& Taddeo, 2010).

$$
\begin{aligned}
S & =\frac{(\mathrm{Ri}-\mathrm{Rmk})}{\mathrm{Dwr}} \\
\text { Dwr } & =\frac{1}{\mathrm{n}} \sqrt{\sum_{1}^{\mathrm{n}} \operatorname{Min}[(\operatorname{Ri}-\mathrm{Rmk}), 0]^{2}}
\end{aligned}
$$

Sendo:

$\mathrm{S}=$ índice de Sortino;

$\mathrm{Ri}=$ retorno médio da carteira;

$\mathrm{Rmk}=$ meta $($ target $)$ de retorno;

Dwr = risco medido pelos desvios abaixo da meta;

$\mathrm{n}=$ número total de retornos.

O downside risk é uma medida de dispersão dos dados abaixo de uma meta selecionada. No cálculo, todos os retornos $\mathrm{R}_{\mathrm{i}}$ acima da meta são zerados, mas estes zeros continuam sendo incluídos na soma. Assim é uma medida distinta do o desvio padrão que retrata a dispersão dos dados em relação a sua média, considerados tanto os resultados abaixo quanto acima da média.

Outra importante métrica é o Alfa de Jensen, que avalia o desempenho dos fundos tendo por base o benchmark fornecido pelo rendimento SML (Security Market Line). O índice mostra a diferença absoluta

$$
E\left(R_{i}\right)=R f+\beta[E(R m)-R f]
$$

Sendo:

$E\left(R_{i}\right)$ - Expectativa de retorno do portfólio;

$\mathrm{Rf}$ - Retorno do ativo livre de risco;

$\beta$ - Beta, que mensura o risco sistemático do portfólio;

$\mathrm{E}(\mathrm{Rm})$ - Expectativa de retorno de uma carteira de mercado.

que existe entre o retorno da carteira com a expectativa de retorno consoante o modelo de precificação de ativos financeiros (CAPM, das iniciais em inglês Capital Asset Pricing Model) desenvolvido por Sharpe (1964) e Lintner (1965).

O CAPM desconsidera o risco específico, uma vez que este pode ser eliminado pela diversificação na montagem de um portfólio. A proposta deste modelo é quantificar o retorno que deve acompanhar determinado grau de risco sistemático (beta) e que não pode ser removido pela diversificação de ativos: 
O coeficiente beta constitui uma medida de sensibilidade de retornos do portfólio em relação ao comportamento de uma carteira de mercado. Quanto maior o beta, maior é o risco, e de acordo com a

$$
E\left(R_{i}\right)-R f=\beta[E(R m)-R f]
$$

$\mathrm{Ou}$ seja, espera-se que para qualquer investimento o excesso de retorno em relação a um ativo livre de risco seja propiciado pelo grau de risco

$$
\mathrm{Yt}=\alpha+\beta \mathrm{Xt}+\varepsilon_{\mathrm{t}}
$$

Sendo:

$\mathrm{Y}_{\mathrm{t}} \mathrm{o}$ excesso de retorno do portfólio em relação à taxa livre de risco;

$\mathrm{X}_{\mathrm{t}} \mathrm{O}$ excesso de retorno de um benchmark em relação à taxa livre de risco;

$\alpha$ é o intercepto;

$\beta$ é a sensibilidade do excesso de retorno do portfólio em relação ao excesso de retorno do benchmark;

$\varepsilon_{\mathrm{t}}$ é o termo de erro da regressão, ou perturbação estocástica, indicando a diferença entre o valor observado e o valor estimado da variável dependente.

A estimativa do coeficiente $\alpha$ da reta de regressão reflete o desempenho do portfólio, mostrando se ele se encontra acima ou abaixo da linha do mercado de títulos, a SML.

\section{Data Envelopment Analysis (DEA)}

Outra ferramenta que vem ganhando destaque na seleção de carteiras eficientes é a DEA (Data Envelopment Analysis). Como se discutiu, no cenário econômico financeiro é necessário estabelecer comparações entre o desempenho de uma carteira com um benchmark. Mas quando existem múltiplos insumos e produtos a análise se torna complexa exigindo modelos de otimização mediante programação linear para encontrar pontos de possíveis modificações em busca de eficiência relativa (Ferreira \& Gomes, 2009).

Charnes, Cooper e Rhodes (1978) salientam que a principal função do DEA é avaliar a eficiência técnica relativa das unidades tomadoras de decisões (DMUs). Estas DMUs podem retratar diferentes fases equação (4) maior é a expectativa de retorno do portfólio.

O Índice Alfa de Jensen (Jensen, 1967) é obtido ao rearranjar a equação (4), subtraindo ambos os lados da equação pela taxa livre de risco $(\mathrm{Rf})$ :

assumido. Em termos econométricos, o Alfa de Jensen pode ser estimado da seguinte forma: do processo produtivo, como a própria empresa, departamentos, segmento de negócios e portfólios de investimentos.

A DEA permite estabelecer a função fronteira de produção, considerando tanto os pontos sobre a curva que delimita a fronteira como os pontos abaixo dela. A solução da programação matemática fornece um score único para cada DMU, que oscilará entre 0 e 1. A DMU é considerada eficiente se o score for 1 , e ineficiente nos demais caso (Ferreira \& Gomes, 2009).

Pode-se trabalhar com modelos de rendimentos constantes de escala (RCE) e de rendimentos variáveis de escala (VRS), expressando os impactos que a variação dos insumos (inputs) pode provocar nos produtos (outputs). O modelo RCE é conhecido com CCR em homenagem aos seus autores (Charnes, et al., 1978), e seu foco está na avaliação objetiva da eficiência global. O modelo VRS ou BCC de autoria de Banker, Charnes e Cooper (1984) calcula a eficiência técnica pura e pode ser expresso da seguinte forma, quando orientado a insumos:

Max:

Sujeito a:

$$
\theta=\sum_{r=1}^{m} u_{r} P_{r k}-u_{k}
$$

$$
\begin{gathered}
\sum_{i=1}^{n} v_{i} I_{i k}=1 \\
\sum_{r=1}^{n} u_{r} P_{r j}-\sum_{i=1}^{n} v_{j} I_{i j}-u_{k} \leq 0, \text { para } j=1, \ldots, s \\
u_{r}, v_{i} \geq 0
\end{gathered}
$$


Sendo:

Prk - quantidade do produto r produzido pela unidade organizacional k;

Iik - quantidade i consumido pela unidade organizacional k;

Prj - quantidade do produto $r$ produzido pela unidade organizacional $j(j=1, \ldots, s)$;

Iij - quantidade do insumo i consumido pela unidade organizacional $\mathrm{j}$;

$\mathrm{r}$ - número de produtos $(\mathrm{r}=1, \ldots, \mathrm{m})$;

$\mathrm{i}$ - número de insumos $(\mathrm{i}=1, \ldots . \mathrm{n})$;

ur - peso do produto $\mathrm{r}$;

vi - peso do insumo i;

uk - escalar.

Vários estudos têm utilizado essa ferramenta de pesquisa operacional no âmbito da análise de gestão estratégica das empresas no país (vide, por exemplo,Vendruscolo, Hoffmann \& Freitas, 2012). Na área financeira são encontrados vários estudos, como por exemplo, o de Lopes, Lima, Dutra \& Saurin (2008) que identificou carteiras capazes de superar o resultado do índice Ibovespa e do índice CDI, em um período de 13 anos. No que diz respeito ao desempenho de fundos de investimentos de renda variável cabe destacar o estudo de Iquiapaza e Fonseca (2012). Os autores utilizaram uma amostra de 329 fundos de investimentos em ações no período pós-crise do subprime e os resultados indicaram que os fundos mantiveram uma relação positiva entre risco e retorno anual.

Versando sobre fundos de renda fixa encontrase o estudo de Macedo, Fontes, Cavalcante e Macedo (2010), que salienta ser o modelo DEA uma importante peça para análise de desempenho. Esta conclusão é, também, ressaltada no trabalho de Rotela, Pamplona e Solomon (2013), segundo os quais a DEA pode fornecer importantes informações para a tomada de decisões dos gestores e na montagem e seleção de portfólios.

Em suma, a Análise Envoltória de Dados oferece novas possibilidades aos analistas do mercado financeiro, com uma metodologia inovadora capaz de transformar a visão dos gestores e investidores e, com isto, aumentar a eficiência da indústria.

\section{MÉTODO DA PESQUISA}

Uma das categorias de fundos de investimentos que ganhou força em seu segmento no período de expansão recente da economia brasileira foi à dedicada aos ativos decorrentes de investimentos imobiliários (FII). Esses fundos são formados por grupos de investidores com o objetivo de aplicar recursos em todo o tipo de negócios de base imobiliária, seja no desenvolvimento de empreendimentos imobiliários ou em imóveis prontos, como edifícios comerciais, shopping centers e hospitais. Do patrimônio de um fundo podem participar um ou mais imóveis, parte de imóveis, direitos a eles relativos, entre outros. O objetivo é conseguir retorno pela exploração de locação, arrendamento, venda do imóvel e demais atividades do setor imobiliário.

Não restam dúvidas de que os fundos de investimentos imobiliários apresentaram um notável crescimento desde 2005, tendo se beneficiado da grande explosão de preços do mercado imobiliário no país (Locatelli, et al., 2017). Essa nova classe de produto financeiro responde por cerca de $2 \%$ da composição total da indústria de fundos do Brasil, estando presente nos portfólios de pessoas físicas e jurídicas. No entanto, não se identificam trabalhos acadêmicos voltados para a análise deste tipo de fundo, e não são divulgados estudos sobre o desempenho relativo vis-à-vis outras aplicações e sobre os riscos envolvidos nesta opção de investimento, temas que constituem o objeto da presente análise.

\section{Caracterização da pesquisa, identificação da} amostra e período de análise

Esta pesquisa é desenvolvida, portanto, na forma metodológica exploratória, e tem por objetivo analisar o desempenho dos FII e identificar aqueles de maior eficiência. Conforme tipologia apresentada por Mattar (1997) é uma pesquisa descritiva/quantitativa, pois utiliza índices, estimativas de um modelo econométrico e técnicas de otimização para explicar o fenômeno estudado.

A identificação dos fundos se deu pela análise do banco de dados do Sistema SI, versão 4.3 da ANBIMA e foram considerados apenas os que apresentavam cotas comercializados na Bolsa de Valores BM\&F Bovespa (atualmente B3). Foram identificados cerca de 280 fundos de investimentos imobiliários abrangendo tanto privados quanto públicos (abertos ao mercado de venda direta), sendo que 131 eram comercializados na B3. Destes restaram 44 Fundos que apresentaram cotações diárias e consistentes ao longo do período considerado, e que foram selecionados como amostra do estudo (relação dos fundos no Anexo).

A pesquisa utiliza informações de setembro de 2013 a agosto de 2016 levantadas no referido banco de dados. Este período, como se sabe, é caracterizado por uma aguda crise política e retração pronunciada da economia, com queda do PIB e na renda real das famílias. Constitui-se, pois, em um período muito adequado para retratar os riscos em aplicações 
financeiras e avaliar aquelas que oferecem maior proteção vis-à-vis os retornos propiciados aos investidores.

\section{Variáveis utilizadas nos diferentes modelos}

Para quantificar a eficiência dos fundos foi adotado como benchmark/meta de mercado a variação do Certificado de Depósito Interbancário (CDI), e como medidas de volatilidade o risco total (empregado no Índice de Sharpe), o downside risk (usado no Índice de Sortino) e o beta (estimado no Alfa de Jensen). Nas estimativas do Alfa de Jensen, além dos retornos dos vários fundos e do benchmark, foi empregada a Selic como proxy da taxa livre de risco.

Para selecionar as variáveis de entradas (inputs) e saídas (outputs) utilizadas no modelo DEA procedeuse uma leitura crítica de trabalhos voltados à análise de desempenho de ativos financeiros no país, tais como Rocha (2013), Lopes et al. (2010), Darwich, Gutierrez e Lopes (2009) e Lopes et al. (2008). Recorreu-se, também, a alguns analistas do mercado para referendar a escolha inicial das variáveis, que de toda a maneira foram condicionadas aos dados disponíveis nos sites especializados e em balanços patrimoniais das empresas. Sobressaíram-se as seguintes variáveis:

- Rentabilidade do fundo, $\mathrm{R}_{\mathrm{FII}}$ (retornos no período selecionado);

- Risco do fundo;

- Custo efetivo da taxa de administração do fundo $(\%$ do custo da gestão pelo investimento);

- Tempo de existência do fundo;

- Quantidade de cotistas;

- Tamanho do fundo (Patrimônio Líquido).

As estimativas do Alfa de Jesen foram obtidos com o uso do E-views 9.0, e as do modelo DEA com o uso do software SDEA BR, sendo este desenvolvido pelo Núcleo de Pesquisa em Eficiência, Sustentabilidade e Produtividade (NESP-UFMG).

\section{DISCUSSÃO DOS RESULTADOS}

Para alcançar os objetivos da pesquisa foram desenvolvidas três etapas. Inicialmente, foram quantificados os índices de desempenho corrigidos pelo risco segundo a perspectiva de Sharpe e de Sortino. Em seguida foram estimadas as regressões na forma sugerida por Jensen. E finalmente foram estimadas as eficiências dos FII de acordo com a abordagem DEA.

\section{Desempenho dos fundos segundo o Índice de Sharpe, Sortino e Alfa de Jensen}

Com os cálculos do Índice de Sharpe e de Sortino é possível estabelecer um paralelo entre o retorno do fundo e sua relação com o mercado (benchmark). O primeiro índice é, conforme se discutiu, padronizado pelo risco total $(\sigma)$ que leva em consideração todos os retornos obtidos no período da análise. Já o segundo enfatiza o risco propiciado apenas pelos resultados que não conseguem superar o benchmark. Assim neste último caso, retornos superiores aos do benchmark são zerados na apuração da volatilidade.

Como os investidores tendem a aplicar recursos com uma visão de retorno de prazo mais longo foi quantificado o desempenho dos quarenta e quatro fundos (44) fundos da amostra fundos, devidamente corrigidos pelo risco, considerando o período como um todo: 36 observações mensais abrangendo de setembro de 2013 a agosto de 2016 (os resultados para toda a amostra podem ser vistos no Apêndice, Tabela A.1). Constatou-se que desempenho dos FIIs corrigido pelo risco foi frustrante: apenas três (03) dos quarenta e quatro (44) fundos se mostraram eficientes tanto em relação ao Índice de Sharpe quanto ao Índice de Sortino: BTG Pactual Fundo de Fundos (BCFF), Campus Faria Lima (FCFL) e o Parque Dom Pedro Shopping Center (PQDP), e eles estão discriminados na Figura 1.

\begin{tabular}{|clllc|}
\hline Ranking & \multicolumn{2}{c}{ Índice de Sharpe } & \multicolumn{2}{c|}{ Índice de Sortino } \\
\hline $1^{\circ}$ & BCFF & 0,1778 & BCFF & 1,6388 \\
$2^{\circ}$ & FCFL & 0,0547 & FCFL & 0,0882 \\
$3^{\circ}$ & PQDP & 0,0047 & PQDP & 0,0075 \\
\hline
\end{tabular}

Figura 1 - Fundos Eficientes no Longo Prazo: setembro 2013 a agosto 2016. Fonte: Tabela A.1 do Apêndice. Elaborado pelos autores, 2018 
A Tabela 1 apresenta os resultados das estimativas do Alfa de Jensen, sendo listados apenas aqueles fundos para os quais o coeficiente $(\alpha)$ se mostrou estatisticamente significativo no nível menor ou igual a $10 \%$. Nesta Tabela os fundos que exibiram coeficientes positivos são considerados eficientes e os de coeficientes negativos são ineficientes, pois os retornos ficaram acima ou abaixo da linha de mercado dos títulos, respectivamente. Os demais fundos não relacionados ficaram na curva de mercado, ou seja, os resultados não permitiram rejeitar a hipótese nula (H0) que estabelece que $\alpha=$ zero.

Tabela 1 - Alfa de Jensen Referente aos FIIs: setembro de 2013 a agosto de 2016*

\begin{tabular}{|lccccc|}
\hline Fundo & Coeficiente & Erro padrão & Estatística t & Prob. Sig. & Desempenho \\
\hline 01 CEOC & $-0,1162$ & 0,0686 & $-1,6937$ & 0,0907 & Ineficiente \\
02 AGCX & 0,1047 & 0,0339 & 3,0857 & 0,0021 & Eficiente \\
03 JSRE & 0,0919 & 0,0498 & 1,8447 & 0,0655 & Eficiente \\
\hline
\end{tabular}

Observação: * Fundos com o coeficiente $\alpha$ comprobabilidade de significância $\leq 10 \%$.

Fonte: Elaborado pelos autores, 2018

Os resultados, também, não foram favoráveis aos FIIs enquanto alternativa atraente para a aplicação de recursos no período analisado. Observa-se que apenas dois fundos superaram os retornos previstos - os Fundos Agências Caixa (AGCX) e JS Real Estate Multigestão (JSRE) - e um não conseguiu apresentar o desempenho corrigido pelo risco - Cyrela Commercial Properties (CECO) (Tabela 1). Pode-se concluir que há aderência dos desempenhos dos FII aos fundamentos do CAPM, sendo que de uma amostra de 44 (quarenta e quatro) fundos, 41 (quarenta e um) deles apresentaram resultados compatíveis com os previstos pelo modelo.

\section{Resultados do modelo DEA}

Nesta seção são analisados os resultados obtidos com o uso do da Análise Envoltória de Dados, tendo sido empregado o modelo VRS orientado para minimizar insumos, conforme especificado no modelo (equação 7). São considerados eficientes os fundos que apresentam score de $100 \%$, sendo estes utilizados como benchmark para os outros fundos se tornarem igualmente eficientes.

Dentre os fundos analisados: 16 (dezesseis) foram classificados como eficientes entre setembro de 2013 e agosto de 2014; 10 (dez) entre setembro de 2014 e agosto de 2015; e 9 (nove) entre setembro de 2015 e agosto de 2016 (Figura 2).

\begin{tabular}{|c|c|c|c|}
\hline $\mathbf{N}^{\mathbf{0}}$ & Set 2013 a Ago 2014 & Set 2014 a Ago 2015 & Set 2015 a Ago 20 16 \\
\hline 01 & HGJH & BCFF & CEOC \\
\hline 02 & FAED & CEOC & THRA \\
\hline 03 & CEOC & THRA & FLRP \\
\hline 04 & THRA & FLRP & MAXR \\
\hline 05 & FLRP & MAXR & PQDP \\
\hline 06 & MAXR & JRDM & JRDM \\
\hline 07 & JRDM & RNGO & RNGO \\
\hline 08 & AGCX & ONEF & ONEF \\
\hline 09 & BMLC & KNCR & FIIP \\
\hline 10 & RNGO & FIIP & \\
\hline
\end{tabular}


Análise da Eficiência dos Fundos de Investimento Imobiliário em um Período de Crise Econômica

\begin{tabular}{|c|c|l|l|}
\hline 11 & ONEF & & \\
\hline 12 & FIIP & & \\
\hline 13 & SAAG & & \\
\hline 14 & SDIL & & \\
\hline 15 & XTED & & \\
\hline 16 & TRLX & & \\
\hline
\end{tabular}

Figura 2 - Fundos classificados como Eficientes pelo DEA Fonte: Elaborado pelos autores.

Similarmente ao observado com as metodologias de Sharpe e de Sortino, verifica-se que poucos fundos conseguiram manter o desempenho positivo ao longo do tempo. Nesta perspectiva, restaram como eficientes em todos os três períodos os
Fundos CEOC, THRA, FLRP, MAXR, JRDM, RNGO, ONEF e FIIP (Figura 2).

Procurou-se analisar a dinâmica dos FII no período analisado, observando se a tendência ocorre na direção de maior ou de menor eficiência (Tabela 2).

Tabela 2 - Ganhos e Perdas de Eficiência dos Fundos: Modelo DEA

\begin{tabular}{|c|c|c|c|c|c|c|c|c|c|}
\hline FUNDO & $\begin{array}{c}09 / 13 \\
a \\
08 / 14\end{array}$ & $\begin{array}{c}09 / 14 \\
a \\
08 / 15\end{array}$ & $\begin{array}{c}09 / 15 \\
\mathrm{a} \\
08 / 16\end{array}$ & $\begin{array}{l}\text { Sentido } \\
\text { da } \\
\text { mudança }\end{array}$ & FUNDO & $\begin{array}{c}09 / 13 \\
\text { a } \\
08 / 14\end{array}$ & $\begin{array}{c}09 / 14 \\
a \\
08 / 15\end{array}$ & $\begin{array}{c}09 / 15 \\
\text { a } \\
08 / 16\end{array}$ & $\begin{array}{l}\text { Sentido } \\
\text { da } \\
\text { mudança }\end{array}$ \\
\hline \multirow{3}{*}{$1 \mathrm{AEFI}$} & 0,9758 & & & & & 0,58 & & & \\
\hline & & 0,862 & & & 23 PRSV & & 0,5614 & & \\
\hline & & & 0,8376 & $\downarrow$ & & & & 0,5949 & $\uparrow$ \\
\hline \multirow{3}{*}{$2 \mathrm{BBFI}$} & 0,3111 & & & 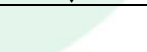 & & 1 & & & \\
\hline & & 0,3158 & & & 24 JRDM & & 1 & & \\
\hline & & & 0,3341 & $\uparrow$ & & & & 1 & \\
\hline \multirow{3}{*}{3 ВBPO } & 0,8706 & & & & & 0,5222 & & & \\
\hline & & 0,7819 & & & 25 ALMI & & 0,5546 & & \\
\hline & & & 0,722 & $\downarrow$ & & & & 0,5222 & \\
\hline \multirow{3}{*}{4 BBRC } & 0,7727 & & & & & 1 & & & \\
\hline & & 0,6786 & & & 26 AGCX & & 0,8635 & & \\
\hline & & & 0,6705 & $\downarrow$ & & & & 0,8742 & $\downarrow$ \\
\hline \multirow{3}{*}{5 HGBS } & 0,3782 & & & & & 0,6446 & & & \\
\hline & & 0,3848 & & & 27 BBVJ & & 0,6151 & & \\
\hline & & & 0,3572 & $\downarrow$ & & & & 0,5389 & $\downarrow$ \\
\hline \multirow{3}{*}{$6 \mathrm{HGJH}$} & 1 & & & & & 1 & & & \\
\hline & & 0,9966 & & & 28 BMLC & & 0,8606 & & \\
\hline & & & 0,8408 & $\downarrow$ & & & & 0,8101 & $\downarrow$ \\
\hline \multirow{3}{*}{7 HGLG } & 0,648 & & & & & 0,5674 & & & \\
\hline & & 0,5696 & & & 29 FLMA & & 0,5713 & & \\
\hline & & & 0,4866 & $\downarrow$ & & & & 0,5276 & $\downarrow$ \\
\hline \multirow{3}{*}{8 HGRE } & 0,4199 & & & & & 1 & & & \\
\hline & & 0,396 & & & 30 RNGO & & 1 & & \\
\hline & & & 0,3338 & $\downarrow$ & & & & 1 & \\
\hline \multirow{3}{*}{9 VRTA } & 0,9595 & & & & & 1 & & & \\
\hline & & 0,7549 & & & $31 \mathrm{ONEF}$ & & 1 & & \\
\hline & & & 0,6985 & $\downarrow$ & & & & 1 & \\
\hline \multirow{3}{*}{10 FAED } & 1 & & & & & 0,6541 & & & \\
\hline & & 0,9261 & & & 32 FVBI & & 0,6568 & & \\
\hline & & & 0,7698 & $\downarrow$ & & & & 0,6234 & \\
\hline
\end{tabular}


Análise da Eficiência dos Fundos de Investimento Imobiliário em um Período de Crise Econômica

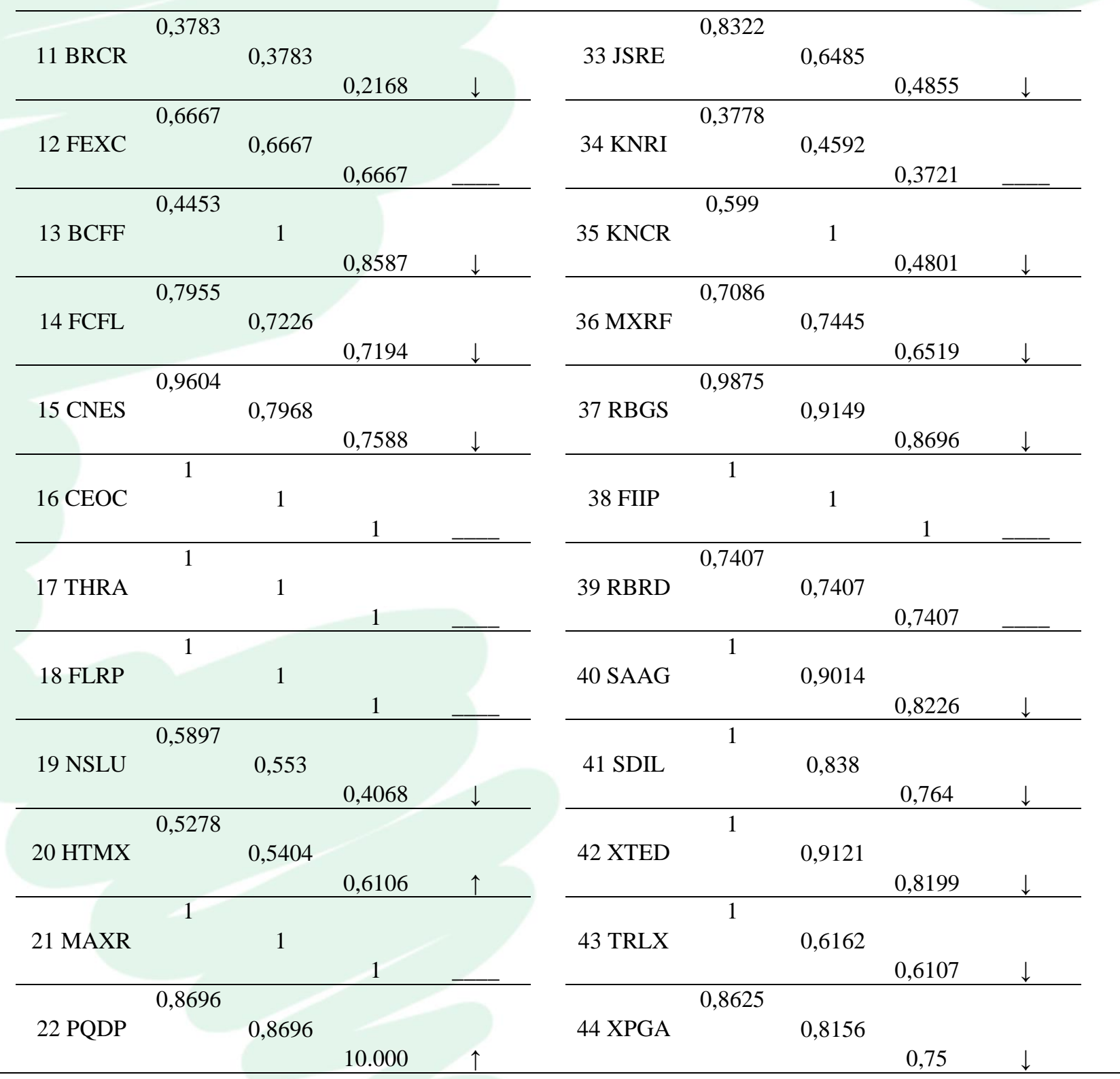

Fonte: Elaborado pelos autores, 2018

Observam-se comportamentos distintos nos vários fundos em relação a ganhos e perdas de eficiência ao longo do período. Contudo, no geral, houve perda de eficiência por parte significativa da indústria, sendo que 28 tornaram-se menos eficientes, 12 não apresentaram mudanças dignas de notas, e apenas 4 elevaram seus níveis de eficiência.

\section{Análise comparativa dos índices de eficiência}

Para finalizar esta seção procurou verificar se as diferentes medidas de eficiência oferecem a mesma hierarquização dos fundos de investimento imobiliários. Para tal, utilizou-se a análise de correlação não paramétrica de Spearman, e os resultados estão apresentados na Tabela 3. 
Tabela 3 - Correlações entre os Diferentes Índices de Eficiência

\begin{tabular}{|llccc|}
\hline & $\begin{array}{c}\text { Índice de } \\
\text { Sharpe }\end{array}$ & $\begin{array}{c}\text { Índice de } \\
\text { Sortino }\end{array}$ & $\begin{array}{c}\text { Alfa de } \\
\text { Jensen }\end{array}$ & DEA \\
\hline Índice de Sharpe & 1,000 & & & \\
Índice de Sortino & $0,991^{*}$ & 1,000 & & \\
Alfa de Jensen & $0,366^{* *}$ & $0,344^{* *}$ & 1,000 & \\
DEA & 0,249 & 0,236 & 0,141 & 1,000 \\
\hline
\end{tabular}

Nota: As correlações não paramétricas foram obtidas aplicando-se o Spearman Rank Correlation.

* Correlação significante no nível de 0,01 .

** Correlação significante no nível de 0,05 .

Fonte: Elaborado pelos autores, 2018

As correlações entre os diversos indicadores de eficiência para os períodos analisados revelam informações interessantes. Verifica-se que os Índices de Sharpe e de Sortino têm uma correlação positiva e coeficiente muito elevado. Em relação às correlações destes dois índices e o Alfa de Jensen, o quadro é distinto. Os índices exibiram, também, uma correlação positiva, porém com coeficientes bem reduzidos. Em relação ao DEA as correlações foram ainda menores e sem significância estatística (Tabela 3).

Em suma, os resultados apresentados indicam que não houve diferença significativa entre a hierarquização fornecida pelos Índices de Sharpe e Sortino, sendo desnecessário o uso de um deles para a seleção de portfólio. Contudo, ao se introduzir na análise uma medida de volatilidade que retrata apenas o risco sistemático (beta), conforme expresso na medida de Jensen, a hierarquização é bastante modificada. E não há compatibilização entre esses diferentes índices e aqueles obtidos pelo modelo DEA.

\section{CONSIDERAÇÕES FINAIS}

O estudo teve por objetivo analisar a eficiência dos Fundos de Investimento Imobiliário empregandose uma amostra de 44 importantes fundos cotados na B3. Foram selecionados como modelos de análise os índices de Sharpe, de Sortino e o Alfa de Jensen, cujo uso é bem documentado na literatura. Além destes, utilizou-se a metodologia de Analise Envoltória de Dados, que tem múltiplas aplicações em medidas de eficiência e vem ganhando espaço nas análises econômico-financeiras. Na DEA foram empregadas, além dos retornos e os riscos, variáveis que refletem a experiência da administradora/gestora decorrente dos anos de existência do fundo, o valor do patrimônio, a taxa de administração e o número de cotistas.

Adotou-se como benchmark de mercado o CDI e como proxy para a taxa livre de risco a Selic. Foram levantados os desempenhos e estimados os riscos de cada fundo utilizando os conceitos de desvio padrão, de downside risk e o beta do fundo. Tendo em vista a elevação da taxa básica de juros e do CDI e a retração do mercado imobiliário no período analisado, os FII apresentaram, em geral, desempenho insatisfatório. Em uma perspectiva de longo prazo (36 meses), apenas três conseguiram superar o benchmark, quer se use o Índice de Sharpe ou de Sortino. Na concepção do Alfa de Jensen, variante do modelo CAPM, considerando o período como um todo, apenas dois fundos se mostraram eficientes por apresentarem retornos acima da linha de mercado de títulos.

A eficiência relativa dos FII foi analisada com maior detalhe com o emprego do modelo DEA. Foi utilizado o modelo DEA VRS, orientado para minimizar insumos, uma vez que este modelo, segundo a literatura resenhada, é o que melhor se adequa à análise de seleção de carteiras. Os resultados obtidos com o modelo de otimização confirmaram os alcançados com os modelos de Sharpe, Sortino e Alfa de Jensen, e revelaram que poucos fundos conseguiram manter o desempenho positivo ao longo do tempo. De forma geral, houve perda de eficiência por parte da amostra desta indústria, sendo que 28 tornaram-se menos eficientes, 12 não apresentaram mudanças dignas de notas, e apenas 4 elevaram seus níveis de eficiência.

Por fim, procurou identificar se as diferentes medidas de eficiência guardam consistência entre si, e se podem ser utilizadas individualmente sem perdas de informações. A análise de correlação não ofereceu respaldo a essa interpretação. Os resultados derivados do Alfa de Jensen e os da DEA, indicaram uma hierarquização de setores distinta daquelas obtidas pelos índices de Sharpe e de Sortino. Diante dos fatos, fica sugerida a importância da utilização de diferentes modelos em situações concretas, pois os resultados podem trazer novos conhecimentos, tanto para os trabalhos acadêmicos quanto para balizar as decisões de investimento. Desnecessário dizer que novas pesquisas devem ser realizadas para validar as conclusões deste estudo, cujos resultados podem contribuir para a tomada de decisão por parte dos gestores e investidores dessa indústria. 
Análise da Eficiência dos Fundos de Investimento Imobiliário em um Período de Crise Econômica

\section{REFERÊNCIAS}

Associação Brasileira de Entidades do Mercado de Capitais [AMBIMA] (2016). Fundos de Investimento. Recuperado de

http://www.anbima.com.br/pt_br/assuntos/fundos-deinvestimento.htm.

Berk, J., Demarzo, P. \& Harford, J. (2009).

Fundamentos de Finanças Empresariais. Porto Alegre: Bookman.

Banker, R. D., Charnes, A.W. \& Cooper, W. W. (1984). Some models for estimating technical and scale inefficiencies in data envelopment analysis,

Management Science, 30(9): 1078-1092.

Charnes, A., Cooper, W. W. \& Rhodes, E. (1978). Measuring the efficiency of decision making units. European Journal of Operational Research, 2(6): 429444.

Damodaran, A. (2016). Investment valuation - tools and techniques for determining the value of any asset. $3^{\text {rd }}$ Edition. New York: John Willey \& Sons Ltd.

Darwich, H., Gutierrez, R. H. \& Lopes, A. L. M. (2009). Seleção de ativos e critérios de alocação de recursos para gestão passiva de carteiras referenciadas ao Ibovespa utilizando Data Envelopment Analysis DEA. Artigo apresentado no XXIX Encontro Nacional de Engenharia de Produção - ENEGEP. Salvador-BA, Brasil.

Eid Jr,,W., Rochman, R. R. \& Taddeo, M. (2010). Medidas de desempenho de fundos considerando risco de estimação. FGV. Recuperado de https://bibliotecadigital.fgv.br/dspace/bitstream/handle/ 10438/15558/Medidas\%20de\%20desempenho\%20de \% 20fundos\%20 considerados\%20riscos\%20de\%20estima $\% \mathrm{C} 3 \% \mathrm{~A} 7 \% \mathrm{C} 3 \% \mathrm{~A} 30$. pdf? sequence $=1$ \&isAllowed=y

Elton, E. J., Gruber, M. J., Brown, S. J.\& Goetzmann, W. N. (2012). Moderna Teoria de Carteiras e Análise de Investimentos. Rio de Janeiro: Elsevier.

Ferreira, C. M. C. \& Gomes, A. P. (2009). Introdução à análise envoltória dos dados. Teoria, modelos e aplicações. Viçosa-MG: Editora UFV.

Iquiapaza, R. A. \& Fonseca. S. E. (2012). Eficiência dos fundos de investimento em ações no período póscrise do subprime: uma aplicação da Análise Envoltória de Dados - DEA. Artigo apresentado em IX Convibra Administração - Congresso Virtual Brasileiro de Administração. Recuperado de http://adm.convibra.com.br
Jensen, M.C. (1967). The Performance of mutual funds in the period 1945-1964, Journal of Finance, 23(2): 389-416.

Lima, F. G. (2015). Análise de Riscos. São Paulo: Atlas.

Lintner, J. (1965). Security prices, risk and maximal gains from diversification. Journal of Finance. 20: 587-616.

Locatelli, R. L., Inês., H M., Lara., J. E. \& Nogueira, F. T. (2017). Real estate market of Brazilian metropolis: sustained growth or speculative bubble? Revista de Administração Mackenzie, 18(2): 211-236.

Lopes, A. L. M., Carneiro. M. L., Schneider, A. B. \& Lima, M. V. A. (2010). Markowitz na otimização de carteiras selecionadas por Data Envelopment Analysis - DEA. Revista Gestão e Sociedade, 4(9): 640-656.

Lopes, A. L. M., Lima, M. V. A., Dutra, A. \& Saurin, V. (2008). Data Envelopment Analysis - DEA como estratégia para seleção de carteiras de investimentos: uma aplicação a 13 anos do mercado de ações brasileiros. Artigo apresentado no XXXII Encontro da Anpad. Rio de Janeiro, Brasil.

Macedo, M., Fontes, P., Cavalcante, G. \& Macedo, H. (2010). Análise do grau de atratividade de fundos de renda fixa: uma abordagem multicriterial da estrutura de oferta utilizando DEA. Revista Contemporânea de Economia e Gestão, 8 (1): 71-82.

Markowitz, H. (1959). Portfolio selection: efficient diversification of investments. New York: Wiley.

Mattar, F. (1997). Pesquisa de marketing: metodologia e planejamento. $4^{\mathrm{a}}$ ed. São Paulo: Atlas.

Rocha, M. D., (2013). Análise de desempenho de fundos de renda fixa no Brasil por meio da análise envoltória de dados (DEA). (Dissertação de Mestrado Profissional em Regulação e Gestão de Negócios). Universidade de Brasília - UnB. Brasília-DF, Brasil.

Rotela, P., Pamplona, E. O. \& Solomon, F. L. R. (2013). Otimização de carteiras: Markowitz associado à análise envoltória de dados. Artigo apresentado no XLVSBPO Simpósio Brasileiro de Pesquisa Operacional, Natal-RN, Brasil. Recuperado de http://www.din.uem.br/ ademir/sbpo/sbpo2013/pdf/arq 0137.pdf

Sharpe, W. F. (1964). Capital asset prices: a theory of market equilibrium under conditions of risk. The Journal of Finance, 19(3): 425-442. 
Análise da Eficiência dos Fundos de Investimento Imobiliário em um Período de Crise Econômica

Sharpe, W. F. (1966). Mutual Fund Performance. The Journal of Business, 39(1): 119-138.

Sortino, F.A. \& Price, L.N. (1994), Performance Measurement in a downside risk framework, Journal of Investing, 3(3): 59-64.

Varga, G. (2001). Índice de Sharpe e outros indicadores de performance aplicados a fundos de ações brasileiros. Revista de Administração Contemporânea, 5 (3): 215-245.

Vendruscolo, B., Hoffmann, V. M. \& Freitas, C. A. (2012). A influência de ferramentas de gestão estratégica e de stakeholders no desempenho de organizações do ramo particular de saúde do Distrito Federal, Revista Ibero-Americana de Estratégia, 11(1): 30-61. 
Análise da Eficiência dos Fundos de Investimento Imobiliário em um Período de Crise Econômica

APÊNDICE

\begin{tabular}{|c|c|c|c|}
\hline SIGLA & FUNDO & SIGLA & FUNDO \\
\hline 01 AEFI & AESAPAR & 23 PRSV & Presidente Vargas \\
\hline 02 BBFI & BB Progressivo & 24 JRDM & Shopping Jardim Sul \\
\hline $03 \mathrm{BBPO}$ & BB Progressivo II & 25 ALMI & Torre Almirante \\
\hline $04 \mathrm{BBRC}$ & BB Renda Corporativa & $26 \mathrm{AGCX}$ & Agências Caixa \\
\hline 05 HGBS & CSHG Brasil Shopping & $27 \mathrm{BBVJ}$ & BB Votorantim JHSF Cidade Jardim Cont.Tower \\
\hline 06 HGJH & CSHG JHSF Prime Offices & 28 BMLC & BM Brascan Lajes Corporativas \\
\hline 07 HGLG & CSHG Logística & 29 FLMA & Continental Square Faria Lima \\
\hline 08 HGRE & CSHG Real Estate & 30 RNGO & Rio Negro \\
\hline 09 VRTA & Fator Verita & $31 \mathrm{ONEF}$ & The One \\
\hline 10 FAED & Anhanguera Educacional & 32 FVBI & VBI FL 4440 \\
\hline $11 \mathrm{BRCR}$ & BTG Pactual Corporate Office & 33 JSRE & JS Real EstateMultigestão \\
\hline $12 \mathrm{FEXC}$ & BTG Pactual Fundo de CRI & $34 \mathrm{KNRI}$ & Kinea Renda Imobiliária \\
\hline $13 \mathrm{BCFF}$ & BTG Pactual Fundo de Fundos & $35 \mathrm{KNCR}$ & Kinea Rendimentos Imobiliários \\
\hline 14 FCFL & Campus Faria Lima & $36 \mathrm{MXRF}$ & Maxi Renda \\
\hline 15 CNES & CENESP & 37 RBGS & RB Capital General Shopping Sulacap \\
\hline $16 \mathrm{CEOC}$ & CyrelaCommercialProperties & 38 FIIP & RB Capital Renda I \\
\hline 17 THRA & CyrelaThera Corporate & 39 RBRD & RB Capital Renda II \\
\hline 18 FLRP & Floripa Shopping & $40 \mathrm{SAAG}$ & Santander Agências \\
\hline $19 \mathrm{NSLU}$ & Hospital Nossa Senhora de Lourdes & 41 SDIL & SDI Logística Rio \\
\hline 20 HTMX & Hotel Maxinvest & 42 XTED & TRX Edifícios Corporativos \\
\hline $21 \mathrm{MAXR}$ & Max Retail & 43 TRLX & TRX Realty Logística Renda I \\
\hline 22 PQDP & Parque Dom Pedro Shopping Center & 44 XPGA & XP Gaia Lote I \\
\hline
\end{tabular}

Figura A1 - Relação dos fundos de investimentos Imobiliários da Amostra. Fonte: Elaborado pelos autores. 
Análise da Eficiência dos Fundos de Investimento Imobiliário em um Período de Crise Econômica

Tabela A.1 - Desempenho dos Fundos de Investimento Imobiliário: setembro de 2013 a agosto de 2016

\begin{tabular}{|c|c|c|c|c|c|c|c|c|c|}
\hline \multirow{2}{*}{$\begin{array}{c}\text { Ranking } \\
1^{\circ}\end{array}$} & \multicolumn{2}{|c|}{ Índice de Sharpe } & \multicolumn{2}{|c|}{ Índice de Sortino } & \multirow{2}{*}{$\begin{array}{c}\text { Ranking } \\
23^{\circ}\end{array}$} & \multicolumn{2}{|c|}{ Índice de Sharpe } & \multicolumn{2}{|c|}{ Índice de Sortino } \\
\hline & BCFF & 0,1778 & BCFF & 1,6388 & & RNGO & $-0,1927$ & HGJH & $-0,2533$ \\
\hline $2^{\circ}$ & FCFL & 0,0547 & FCFL & 0,0882 & $24^{\circ}$ & FVBI & $-0,2088$ & FVBI & $-0,2584$ \\
\hline $3^{\circ}$ & PQDP & 0,0047 & PQDP & 0,0075 & $25^{\circ}$ & BBPO & $-0,2216$ & KNCR & $-0,2667$ \\
\hline $4^{\circ}$ & FAED & $-0,0358$ & FAED & $-0,0496$ & $26^{\circ}$ & BRCR & $-0,2328$ & BBPO & $-0,2750$ \\
\hline $5^{\circ}$ & FLRP & $-0,0585$ & FLRP & $-0,0822$ & $27^{\circ}$ & HGRE & $-0,2362$ & RBRD & $-0,2823$ \\
\hline $6^{\circ}$ & MAXR & $-0,0866$ & MAXR & $-0,1172$ & $28^{\circ}$ & KNCR & $-0,2389$ & HGRE & $-0,2887$ \\
\hline $7^{\circ}$ & FLMA & $-0,0965$ & MXRF & $-0,1242$ & $29^{\circ}$ & RBRD & $-0,2493$ & BRCR & $-0,3017$ \\
\hline $8^{\circ}$ & MXRF & $-0,1013$ & NSLU & $-0,1346$ & $30^{\circ}$ & FIIP & $-0,2590$ & BBVJ & $-0,3042$ \\
\hline $9^{\circ}$ & NSLU & $-0,1044$ & FLMA & $-0,1472$ & $31^{\circ}$ & BBVJ & $-0,2685$ & BMLC & $-0,3168$ \\
\hline $10^{\circ}$ & BBRC & $-0,1133$ & BBRC & $-0,1531$ & $32^{\circ}$ & BMLC & $-0,2806$ & ALMI & $-0,3276$ \\
\hline $11^{\circ}$ & HGBS & $-0,1358$ & HGBS & $-0,1701$ & $33^{\circ}$ & ALMI & $-0,2900$ & SDIL & $-0,3347$ \\
\hline $12^{\circ}$ & ONEF & $-0,1386$ & ONEF & $-0,1750$ & $34^{\circ}$ & SDIL & $-0,2907$ & HTMX & $-0,3504$ \\
\hline $13^{\circ}$ & AGCX & $-0,1398$ & HGLG & $-0,2072$ & $35^{\circ}$ & HTMX & $-0,3006$ & FIIP & $-0,3556$ \\
\hline $14^{\circ}$ & HGLG & $-0,1476$ & CEOC & $-0,2111$ & $36^{\circ}$ & CNES & $-0,3482$ & BBFI & $-0,3747$ \\
\hline $15^{\circ}$ & SAAG & $-0,1582$ & THRA & $-0,2146$ & $37^{\circ}$ & BBFI & $-0,3542$ & XPGA & $-0,3788$ \\
\hline $16^{\circ}$ & THRA & $-0,1652$ & SAAG & $-0,2159$ & $38^{\circ}$ & XPGA & $-0,3610$ & CNES & $-0,3812$ \\
\hline $17^{\circ}$ & CEOC & $-0,1692$ & AGCX & $-0,2180$ & $39^{\circ}$ & VRTA & $-0,4383$ & XTED & $-0,4540$ \\
\hline $18^{\circ}$ & AEFI & $-0,1768$ & JSRE & $-0,2199$ & $40^{\circ}$ & RBGS & $-0,4609$ & VRTA & $-0,4615$ \\
\hline $19^{\circ}$ & KNRI & $-0,1823$ & FEXC & $-0,2240$ & $41^{\circ}$ & JRDM & $-0,4711$ & JRDM & $-0,4698$ \\
\hline $20^{\circ}$ & JSRE & $-0,1823$ & AEFI & $-0,2257$ & $42^{\circ}$ & XTED & $-0,4718$ & PRSV & $-0,4701$ \\
\hline $21^{\circ}$ & FEXC & $-0,1848$ & KNRI & $-0,2270$ & $43^{\circ}$ & PRSV & $-0,4816$ & RBGS & $-0,4793$ \\
\hline $22^{\circ}$ & HGJH & $-0,1925$ & RNGO & $-0,2527$ & $44^{\circ}$ & TRXL & $-0,6157$ & TRXL & $-0,5607$ \\
\hline
\end{tabular}

Fonte: Elaborado pelos autores. 